\title{
Surveillance and Epidemiology of Dengue in Medellín, Colombia from 2009 to 2017
}

\author{
Colin M. Warnes, ${ }^{1}$ Eduardo Santacruz-Sanmartín, ${ }^{1}$ Fausto Bustos Carrillo, ${ }^{2}$ and Iván Darío Vélez ${ }^{1 *}$ \\ ${ }^{1}$ Programa de Estudio y Control de Enfermedades Tropicales (PECET), Universidad de Antioquia, Medellín, Colombia; ${ }^{2}$ Sustainable Sciences \\ Institute (SSI), San Francisco, California
}

\begin{abstract}
Dengue is the most prevalent arthropod-borne viral disease in humans, primarily transmitted by the Aedes aegypti mosquito. We conducted a descriptive analysis of dengue cases from 2009 to 2017 in Medellín, Colombia, using data available from the Secretariat of Health. We analyzed the burden of outbreak years on the healthcare system, risk of cases exhibiting severe illness, potential disease surveillance problems, gender and age as risk factors, and spatiotemporal patterns of disease occurrence. Our data consisted of 50,083 cases, separated based on whether they were diagnostic test negative, diagnostic test positive (primarily IgM ELISA), clinically confirmed, epidemiologically linked, or probable. We used dengue incidence to analyze epidemiological trends between our study years, related to human movement patterns, between gender and age-groups, and spatiotemporally. We used risk to analyze the severity of dengue cases between the study years. We identified human movement could contributed to dengue spread, and male individuals (incidence rate: $0.86 ; 95 \% \mathrm{Cl}: 0.76-0.96$ ) and individuals younger than 15 years (incidence rate: $1.24 ; 95 \% \mathrm{Cl}$ : 1.13-1.34) have higher incidence of dengue and located critical parts of the city where dengue incidence was high. Analysis was limited by participant diagnostic information, data concerning circulating strains, and a lack of phylogenetic information. Understanding the characteristics of dengue is a fundamental part of improving the health outcomes of atrisk populations. This analysis will be useful to support studies and initiatives to counteract dengue and provide context to the surveillance data collected by the health authorities in Medellín.
\end{abstract}

\section{INTRODUCTION}

Dengue virus (DENV) (genus Flavivirus, family Flaviviridae) is a major worldwide public health concern, particularly in developing countries, urban areas, and temperate climates where its vector, Aedes aegypti, is primarily found. ${ }^{1}$ Dengue virus consists of four serotypes (1-4), each antigenically different but all displaying similar clinical characteristics, termed dengue. ${ }^{2}$ After an initial infection, persons are at risk of a more severe secondary infection by a different serotype. ${ }^{3}$ Up to 3.83 billion individuals are at risk worldwide, mainly in Asia followed by the Americas and Africa, and less significantly in the Australia/Pacific Island area and in Europe. ${ }^{4}$ The danger of dengue is exacerbated by issues with medical care in poorer countries; if patients do not obtain proper care, a manageable disease can become deadly. ${ }^{1}$ Regular epidemiological analysis is important to implement targeted public health programs, analyze the different characteristics of dengue geographically and temporally, and find gaps in data and our understanding of the disease.

Global efforts are continuous in the fight against dengue; however, progress has been limited. There are neither effective antiviral agents nor licensed vaccines in widespread use, with care being focused on stabilizing the patient within a hospital setting. Several vaccine clinical trials are in progress, and although some are promising, a comprehensive solution has been elusive. ${ }^{5,6}$ A changing climate, increased global movement, and urban expansion have permitted the vector's spread. ${ }^{7-11}$ The problem is exacerbated by the lack of efficient and affordable diagnostic tools. PCR is the gold standard, but it is expensive and only accurate between the second and sixth day of the acute period. ${ }^{12,13}$ ELISAs against the nonstructural protein 1 (NS1) and for antibodies such as IgG and $\lg \mathrm{M}$ are other accurate ways to diagnose dengue virus infections in the acute and convalescent phases, respectively, but

* Address correspondence to Iván Darío Vélez, Programa de Estudio y Control de Enfermedades Tropicales (PECET)-SIU, Calle 62 N 52-59, Medellin 050010, Colombia. E-mail: ivan.velez@udea.edu.co
NS1 ELISA access is limited, and IgG and IgM can cross-react with antibodies to other arboviruses, rendering it difficult to come up with standardized methods. ${ }^{13-16}$ Others employ less common techniques such as neutralizing assays, inhibition ELISAs, and IgM antibody capture enzyme-linked immunosorbent assays (MAC-ELISAs), with similar limitations. ${ }^{17}$ The lack of reliable and cost-efficient assays causes health systems to lean on incomplete data, and they must be analyzed carefully to generate hypothesis as to which factors that lead to dengue must be prioritized, especially in countries with less resources.

Colombia, with a history of being severely affected by dengue, recently experienced outbreaks in 2010 and 2016, resulting in 147,670 and 103,822 reported cases, respectively. ${ }^{18,19}$ Currently, all four serotypes co-circulate within the country. ${ }^{20}$ Outbreak years are defined as the occurrence of cases (instances of illness) in excess of what would normally be expected. ${ }^{21}$ They are characterized by high rates of disease, exceeding the typical burden of disease in the areas afflicted by the outbreak and can strain medical care systems. Medellín is the second largest metropolitan area in Colombia, with a population of approximately 2.5 million people. ${ }^{22}$ Medellín is divided between 16 comunas, urban districts within the city, and five corregimientos, rural areas outside the center of the city. ${ }^{23}$ Medellín was classified as a hyperendemic municipality as of the year 2013. Surveillance is conducted by the Secretariat of Health in Medellín, and the city was labeled as dengue hyperendemic by the country surveillance system as of $2013 .^{24}$ Every single dengue case must be reported to the Sistema de Vigilancia en Salud Pública (SIVIGILA), the national surveillance system tasked with following dengue cases, tracking them clinically and confirming them through laboratory results. Lee et al. ${ }^{25}$ found the cost of dengue in Latin America to be, on average, $\$ 307$ per case, whereas Lee et al. identified Colombia's dengue burden to be higher than similar other countries, such as Thailand and Vietnam. ${ }^{26}$ Because of its severity and adaptability, dengue presents a problem and huge cost to Medellín.

In a large city like Medellín, outbreaks can begin and spread rapidly through a dense population, making it imperative to 
implement effective interventions. We conducted a descriptive analysis of dengue in Medellín, presenting the cases by year, epidemiological period, gender, age, and place of residence (comuna or corregimiento), using data from SIVIGILA across 2009-2017. We analyze the extent to which outbreak years add greater burden to the medical system, potential problems with the surveillance system, gender and age as risk factors, and how the disease moves spatiotemporally to support future advancements in public health and scientific research.

\section{MATERIALS AND METHODS}

Epidemiological surveillance of dengue in Medellín and related data sets. In Medellín, the dengue public health routine surveillance system is conducted by public health institutions, such as hospitals, community health centers, and surveillance programs, referred to as "Instituctiones Prestadoras de Salud" (IPS). These institutions notify government organizations such as the Municipal Secretary of Health and the Colombian Ministry of Health and Social Protection through SIVIGILA. All cases that meet the clinical dengue case criteria are designated as probable, and IPSs confirm infections through laboratory testing, primarily testing for IgM antibodies against dengue virus but also other ELISA and PCR methods within their own facilities. The type of test used was not included in the SIVIGILA data set, preventing us from analyzing it. To estimate incidence, we combined probable and laboratory-confirmed cases.

In the SIVIGILA database, between 2009 and 2010, cases were reported as either 1) classic dengue, an acute onset of high fever 3-14 days after the bite of an infected mosquito (presenting with frontal headache, retro-orbital pain, myalgias, arthralgias, hemorrhagic manifestations, rash, and low white blood cell count) or 2) severe dengue when symptoms were more pronounced (dengue with complications, dengue hemorrhagic fever, and dengue shock syndrome). ${ }^{27}$ In 2010, the Secretariat of Health in Medellin standardized the process of diagnosing dengue cases by amending their diagnostic process to categorically follow 2009 WHO and Pan American Health Organization (PAHO) guidelines. Thereafter, cases in Medellín were diagnosed as probable using $\mathrm{PAHO}$ criteria for dengue: a fever and two of the following symptoms: nausea/ vomiting, exanthema, headache/retro-orbital pain, myalgia and arthralgia, petechiae or positive tourniquet test, and leukopenia. ${ }^{27}$ Dengue cases were then classified based on three distinct severity criteria: dengue without warning signs (D), dengue with warning signs (DWS), and Severe Dengue (SD). Because the transition to a different reporting system took place in 2010, cases from 2009 do not have a clinical classification in our database and only a proportion of cases in 2010 were clinically classified. Cases were categorized as laboratorynegative, laboratory-confirmed, clinically confirmed, epidemiologically linked, or probable. For the purpose of our analysis, we only looked at laboratory-confirmed, laboratory-negative, and laboratory-unconfirmed cases, where laboratoryunconfirmed is defined as probable, clinically confirmed, or epidemiologically linked cases. Classification as probable is defined as a case that is most likely dengue but does not meet the pronounced features of the clinical classification criteria. A clinically confirmed case more definitively meets classification criteria. The database is cleaned regularly through city- managed quality control methods that eliminated registrations that are duplicates, repeated, and contained errors when the information was uploaded.

Cases were further categorized as imported, exported, foreign, and local. Imported is defined as an individual residing in Medellín who was infected with dengue virus outside the city elsewhere in Colombia or outside the country 14 days before illness onset; exported is defined as an individual who was in Medellín 14 days before illness onset but resides elsewhere in Colombia or outside the country, reported through other municipalities; foreign is defined as an individual who was infected and resides outside the city but was admitted to a hospital in Medellín; and local is an individual who was infected and resides within the city. The data set provided the suspected location where the illness began and whether the patients reside within or outside the city. We categorized cases into two age-groups based on a cutoff of 15 years of age because we assumed children younger than 15 years would be less mobile and stay close to home more so than cases who are at least 15 years old. This age categorization was performed for the purpose of our research and is not a standard distinction within the Ministry of Health in Medellín.

We compared the number of cases during each epidemiological period, weeks of the year organized to account for changing epidemiological cycles, with the average number of cases during standard epidemiological periods. This allowed us to visualize outbreaks as outliers and distinguish standard epidemiological periods that saw greater numbers of cases than normal. As we related the incidence of cases to the different epidemiological periods, we kept in mind the periods of the rainy season, which was defined by Moreno et al. ${ }^{28}$ at the Civil Engineering Department and Universidad Nacional de Colombia.

The yearly population data used to calculate incidence rates (number of new dengue cases per 100,000 population) were obtained from the Departamento Administrativo Nacional de Estadistica.

Statistical analysis. Statistical analyses were conducted in $R$ version 3.5.1 and Microsoft Excel 2019 version 16.26 (Microsoft Corp., Redmond, WA). The incidence rate was calculated by dividing the number of new dengue cases over the population of the group of interest per 100,000 individuals (incident cases/population $\times 100,000$ ). The coefficient of variation (CoV), a standardized measure of variability, was calculated by dividing the standard deviation by the mean of cases per year and multiplying it by 100 (standard deviation/ mean $\times 100$ ). Risk was calculated based on the number of cases classified in each category over the total classified cases that year (dengue classification/total dengue cases).

Ethical considerations. No human subjects research was conducted. A de-identified data set was used, with all patients being labeled with anonymous codes. The Secretarait of Health of Medellín, Colombia, published the SIVIGILA data set publicly, and they are the sole party able to link the data to personally identifiable information, which they did not do for this study. The analysis was conducted on a database of cases meeting the clinical criteria for dengue with no intervention or modification of biological, physical, psychological, or social variables. This investigation is considered safe and is in accordance with Resolution 8,430 of 1993 of Colombia. 


\section{RESULTS}

Trends of disease incidence and diagnosis in Medellín, Colombia. Between years 2009 and 2017, a total of 50,083 cases of dengue were recorded by the Secretariat of Health in Medellín, Antioquia. Cases were diagnosed either clinically by an attending medical professional or confirmed by laboratory tests.

Cases in Medellín were reported year-round and were more pronounced between May and November. The highest annual incidence rates per 100,000 population were recorded in 2016 with 656.37 and in 2010 with 653.81. Because of high numbers of cases and incidences in these years, we will refer to them as outbreak years (Figure 1). Outbreak years are a definitive characteristic of dengue disease, and understanding their trends can help lift the heavy burden they weigh on local health systems.

Overall, the annual incidence ranged from 26.70 to 656.37 cases per 100,000 population (median $87.99 / 100,000$ ). The CoV over the 9 years was 1.29 annually and 1.49 divided by epidemiological period in each year. Looking exclusively at the two outbreak years, there was a CoV of 0.60 and when omitting outbreak years, the CoV was 0.07 . We calculated the CoV to understand how big of a change in cases' regular years have the outbreak years.

During outbreak years, the case incidence was high throughout the year but more focused between May and September. In non-outbreak years, the number of cases increased during the rainy season and toward the end of the year. The majority (22/32) of epidemiological periods above 158.39, the average number of cases during standard epidemiological periods (red line on Figure 1), took place during the rainy season. The lowest incidence almost every year was within the dry season, 2015 being the exception, where the lowest incidence occurred in May, the first month of the rainy season. None of the epidemiological periods that took place during outbreak years decreased below the average number of cases during standard epidemiological periods. There was a noticeable drop-off of cases after outbreak years: 20102011 observed a decrease of $95.6 \%$ and 2016-2017, a decrease of $88.4 \%$. Toward the end of outbreak years, there was a constant drop-off in cases starting from that year's maximum, extending slightly into the start of the next year and then flattening out.

Severity criteria was separated by year and then analyzed over the total number of positive cases clinically categorized to provide an understanding of the severity and clinical outcomes per year. The risk of $D$ and DWS trended opposite throughout our study period. Between years 2010 and 2014, cases maintained around a $90 \%$ risk of $D$ in the cases until 2014 , where it then drops to below $60 \%$, and the risk of DWS cases increased above $40 \%$. The risk of all dengue cases that were SD was higher in the first half of our study period, between 2010 and 2013 and between 1 and $2 \%$ of cases and then decreased to 1\% after 2014 (Figure 2) (Supplemental Table S2).

Cases were categorized as laboratory-negative, laboratoryconfirmed, and laboratory-unconfirmed (Figure 3). In 2009, data on only laboratory-positive and laboratory-unconfirmed cases were provided by the city. Looking exclusively at outbreak years, 2016 was supported by laboratory testing $64.4 \%$ of the time: $20.2 \%$ recorded as negative and $44.2 \%$ as positive, whereas in 2010 , the number of cases tested in the laboratory was dramatically lower, at only $38.4 \%: 4.5 \%$ recorded as negative and $33.9 \%$ as positive. After 2010 , there was a distinct increase in the percent of cases tested in the laboratory, reaching $60 \%$ and only lowering below in 2017 , a year of very few cases. During outbreak years, the cases that were found to be laboratory negative were, without exception, lower than standard years, which ranged from a low of $22.2 \%$ negative cases in 2011 to a high of $52.8 \%$ negative cases in 2017.



FigurE 1. Absolute numbers of dengue cases by epidemiological period in Medellin, Colombia, 2009-2017. Blue represents the rainy season and orange the dry seasons. This figure appears in color at www.ajtmh.org. 



\section{Dengue without Warnings Signs (D) Dengue with Warning Signs (DWS) Severe Dengue (SD)}

FIGURE 2. Dengue severity over total classified cases in Medellin, Colombia, 2009-2017. Cases were divided by three different criteria of severity: dengue without warning signs (D), dengue with warning signs (DWS), and severe dengue (SD). Cases were then separated by year, and risk was calculated by dengue classification over total classified cases at a $95 \% \mathrm{Cl}$. This figure appears in color at www.ajtmh.org.

Dengue trends based on geographic origin, age, and gender. The geographical origin of patients with dengue varied over time, and we conducted this analysis to gain insights into the imported and exported cases in Medellín, CO. Since 2010, the majority of dengue cases originated in Medellín, where the individuals also resided (MDE/Medellín [MDE]) (Table 1). The second largest category of cases was individuals who resided in Medellín but were infected outside the city, defined as "imported" cases (other/MDE). The percentage of cases that were imported increased above $1 \%$ in 2011 and never dropped below. The percentage of imported cases reached its highest in 2015 and dropped off slightly in 2016 and 2017, but both still stayed higher than any of the years before. Patients who resided outside Medellín but were infected inside, known as "exported" cases (MDE/other), were recorded starting in 2010, reaching a high of $2.2 \%$ in 2016 and a low of $0.5 \%$ in 2017 , generally hovering around $1 \%$. The percent of cases infected and residing outside Medellín (other/ other) only had data in 2009 and between 2015 and 2017, only ever increased above $0.5 \%$ during 2016, an outbreak year, reaching $1.1 \%$ (Table 1 ).

The percent split between dengue cases below and above 15 years of age was consistent throughout the years, not fluctuating significantly (Table 2). Every year, the incidence rate was higher for those younger than 15 years, demonstrating that children have higher susceptibility to dengue in Medellín.
We also analyzed differences in dengue cases and incidence between genders. Overall, male individuals had a higher incidence of contracting dengue than female individuals (Table 2). In most of the years, male individuals had a higher percent of cases and incidence. However, female individuals had a slightly higher percentage of the total cases during the 3 years of highest reported dengue cases, 2015 and the two outbreaks, 2010 and 2016. In addition, they had a higher incidence in both 2015 and 2016.

Spatial distribution of dengue among the different comunas and corregimientos. Spatial analysis can help define areas that can be later monitored and targeted to reduce dengue. We measured incidence in the Aburra Valley, the valley within which Medellín is located, annually (Figure 4). Certain patterns were observed based on the comuna in which cases resided. Areas in the east of the city-Popular, Santa Cruz, Manrique, Aranjuez, Villa Hermosa, Buenos Aires, and La Candelaria-had above average incidence every year, apart from 2011. This was exemplified by years 2012 and 2014-2017.

Before, during, and after outbreaks, similar areas experienced high levels of incidence compared with the rest of the city (Table 2) (Supplemental Figure S2) (Supplemental Table S1). In the 2010 outbreak, the cases were focused on the northeast/east and southwest/west sides of the city, particularly around San Javier and Aranjuez. Areas such as Poblado and Robledo kept relatively lower incidence during that period. 


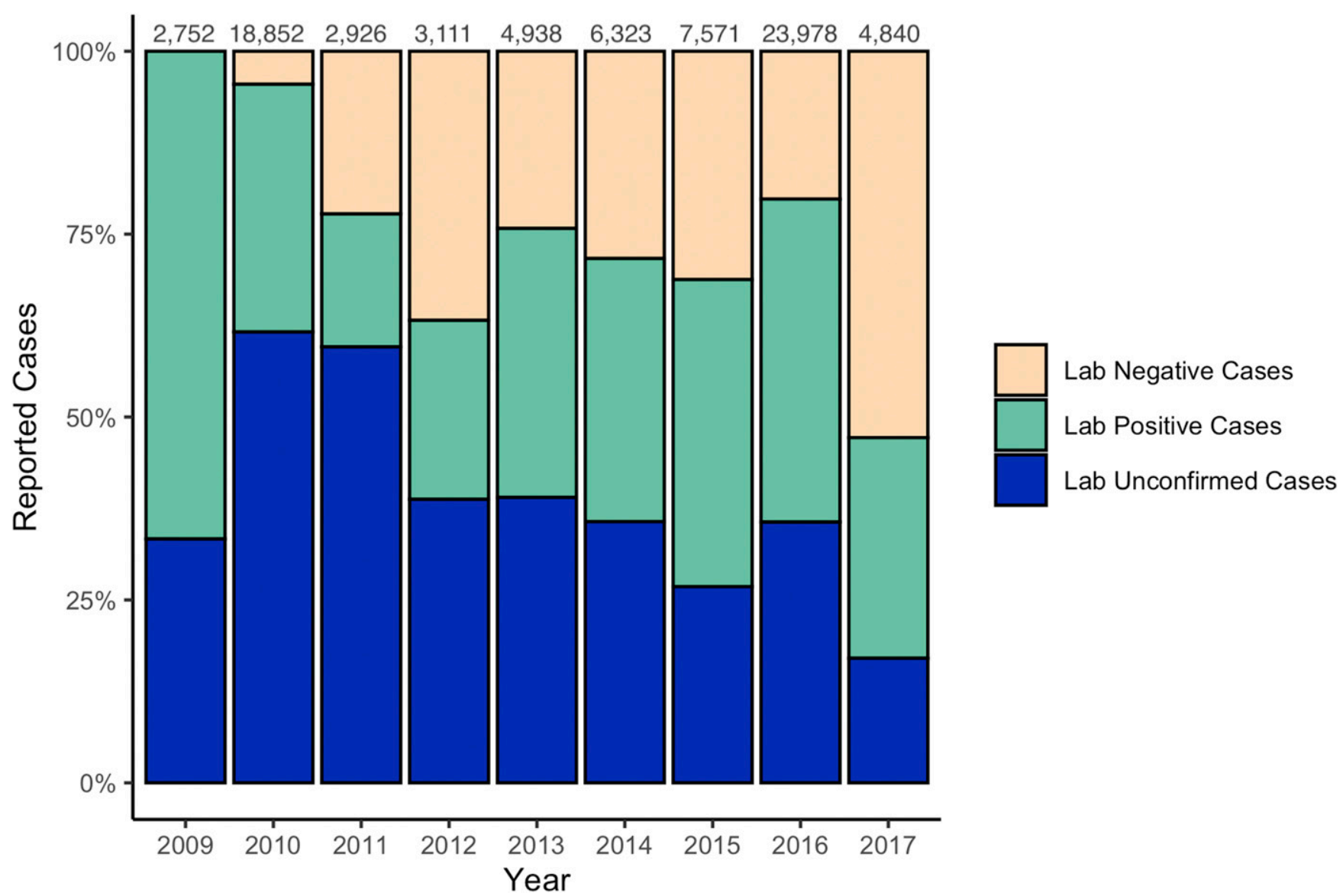

FIGURE 3. Dengue cases by type of confirmation in Medellin, Colombia, 2009-2017. Cases were divided as unconfirmed possible dengue cases, laboratory-confirmed positive or negative for dengue infection, 2009-2017. Cases that were confirmed clinically, epidemiologically linked, or considered probable were classified together as laboratory-unconfirmed cases. The total number of potential cases every year is presented at the top of the graph and includes cases that were laboratory-negative. This figure appears in color at www.ajtmh.org.

In 2016, the outbreak was more focused on the eastern side of the city, specifically around Villa Hermosa, La Candelaria, and Buenos Aires, generating a disproportionately higher level of incidence, 1,612, 1,306, and 1,346 respectively. These three comunas essentially doubled the average incidence in the city during the 2016 outbreak at 656.37 . With spatial analysis such as what we provided, areas can be categorized as generally having higher incidence and residents can be considered more vulnerable.

Dengue incidence was contained more in the center of the valley, distributing itself in higher numbers among the comunas, rather than the corregimientos. Villa Hermosa had the highest incidence for three of the years and levels above the average for six of the 8 years, demonstrating particularly high incidence regularly. Buenos Aires, a bordering comuna, followed closely, generally being among the highest in incidence every year and setting the upper limit in 2012, 2015, and 2016. The corregimientos fluctuated more than the comunas and had outlying years in 2011 with San Sebastian de Palmitas, 2012 with Santa Elena, and 2013 and 2016 with Altavista, respectively.

When the maps were scaled to the range of all given incidence rates throughout the years, outbreak years control the upper limit and stand out very apparently (Supplemental Figure S1). Years 2013 and 2014 were distinct among standard years with a distribution of high incidence that resemble levels of outbreak years but, nevertheless, not surpassing their averages. In years following outbreaks, such as in 2011 and 2017, there was a distinct drop in incidence. Visualizing the large increase in cases provides a reference as to the impact it has on the city and also highlights areas most at risk during outbreak years.

\section{DISCUSSION}

Dengue is considered endemic in Medellín, Colombia, and creates a heavy burden on the Medellín public health system, one that already encounters a variety of diseases because of its tropical climate. ${ }^{29,30}$ Here, we analyze the extent to which outbreak years add greater burden on the medical system, the age and gender as risk factors, and how the disease moves spatiotemporally. Our study provides support to public health initiatives, whether government or non-governmental organization (NGO), in making better decisions on the direction of their project to guarantee broader impact.

Our data consist of the years 2009-2017, where 2010 and 2016 are considered dengue outbreak years, the occurrence of cases of disease in excess of what would normally be expected. $^{21}$ Outbreak years created an especially severe burden on the public health system, with incidence rates as 




high as 656.37 per 100,000 population in 2016 , more than tripling the average incidence rate of 203.69 and more than eight times the average incidence of 74.72 during standard years, years where outbreaks did not occur. There is no consensus on how to predict when outbreaks will occur, and they are often characterized by the prevalence of a serotype that may be different from the previous outbreak. ${ }^{31-34}$

Cases in Medellín are diagnosed as probable using the PAHO standards for dengue. Of probable cases, $56.6 \%$ were taken to be laboratory tested, most commonly for lgM using the ELISA assay. However, without laboratory support, it can be difficult to discern whether the cases were accurately diagnosed, especially in adults who tend to present less severe symptoms. ${ }^{35}$ In fact, we saw that on average, $39.2 \%$ of the cases tested in the laboratory were negative. If we were to extrapolate this to all probable cases, there would be a decrease by up to 3,062 cases in 2016 , the year with the highest number of cases.

Dengue cases can be difficult to discern clinically from other viruses such as influenza, Zika, chikungunya, and yellow fever, leading to mis-categorization. ${ }^{36,37}$ A study conducted in the comuna San Javier in Medellín recruited patients solely based on whether they had fever onset; $9.3 \%$ of the patients were confirmed to have had dengue, although only $4.1 \%$ of said patients were actually diagnosed with dengue by the physician. ${ }^{19}$ A study in Managua, Nicaragua, focused on the percentage of dengue cases who were undiagnosed or misdiagnosed while following the PAHO clinical diagnostic standards. They found that $63.5 \%$ of suspected cases were seropositive for dengue, whereas only $8.1 \%$ were suspected clinically, demonstrating the difficulties in capturing dengue patients. ${ }^{38}$

Diagnosing cases using IgM ELISA is the most common technique used in Medellín, Colombia, because it is the most efficient and cost-effective technique available. However, Sangamuang et al. ${ }^{39}$ found that the IgM ELISA had $70 \%$ sensitivity and $80 \%$ specificity, which can distort the disease numbers. For example, in 2016, there were a total 14,145 cases tested in the laboratory: 4,428 were confirmed as laboratory negative and 9,717 as positive. Up to 1,331 cases could have been false negative and 1943 could have been false positive, misdiagnosed as dengue. In addition, IgM diagnostic assays for dengue virus infection can cross-react between other flaviviruses, such as yellow fever and Zika, distorting data. ${ }^{40-42}$ There is a need for a major breakthrough in the dengue field to allow developing countries the ability to diagnose their patients with utmost confidence. Nevertheless, the PAHO diagnostic system is effective overall, where most cases were correctly diagnosed, confirmed by the available diagnostic methods most of the time in six of our nine study years. The molecular limitations are clear, and the field must continue to work to address these problems to maximize the effectiveness of public health programs.

To understand the severity of dengue cases in Medellín, Colombia, we looked at the risk of being classified as D, DWS, and SD per year. Until 2014, we observed a high risk of D, around $90 \%$. After 2014, which is the period of the highest dengue incidence, apart from the 2010 outbreak, D risk began to drop and DWS risk increased from around $10 \%$ to around $40 \%$. SD cases did not fluctuate very much and were highest before 2014. This indicated that there was potentially higher degree of severity in those final 3 years, as opposed to 




between 2010 and 2014. This increase in severe cases could be linked to a change in the predominant serotype circulating, leading to symptoms facilitated by antibody dependent enhancement or the emergence of a more severe serotype..$^{2,36}$ This could also relate to improved access to care and an improvement in clinical diagnosis for more SD patients in Medellín. This is plausible because in Colombia, there has been momentum since 2002 to improve accreditation within the hospital system and expand access. ${ }^{43}$ Improvements in access to hospitals might encourage dengue patients to seek care, a trend observed in Cali, Colombia. ${ }^{44,45}$

In recent years, Colombia has improved its transportation system throughout the country, and crime and violence have gone down, causing Colombia's tourism to increase and potentially the spread of dengue. ${ }^{46,47}$ In Asia, Tien et al. demonstrated increased air travel playing a large role in the dispersal of dengue virus, and similar conclusions were found in Brazil, which also demonstrated the additional role of the development of roads around a city. ${ }^{48,49}$ In Medellín, imported cases could have played a role in the 2016 outbreak, where in 2015 , we observed the highest percentage of imported cases at $6.7 \%$ and in 2016, the highest total number of imported cases, reaching $4.5 \%$ of all cases, compared with previous years where it ranged from 0.6 to $3.8 \%$ (Table 1). Increased cases due to imported diseases can be mitigated by citywide policies such as preparing rapid responses in hospitals for imported cases, informing travelers how to avoid dengue and respond to symptoms appropriately, and controlling vector populations in more prominent tourist areas.

Our data also suggested that children younger than 15 years and male individuals had a higher incidence rate of dengue. In Brazil, data supported children being more susceptible to dengue, where children younger than 10 years had a higher incidence of hospitalization. ${ }^{50}$ However, other studies contradict this statement, where in Hong Kong, individuals older than 21 years; in Dhaka, Bangladesh adults; and in Yucatan, Mexico adolescences between 15 and 19 years carried the highest incidence, whereas in Argentina, no correlation was found. ${ }^{51-53}$ Reasons for greater dengue in children in Medellín could stem from not having previously developed postsecondary infection immunity or having parents who are attentive to the dangers of dengue, leading to greater chance of reporting dengue. ${ }^{35}$ Male individuals had a higher incidence of dengue than female ones in Medellín; however, it is notable that female individuals had a slightly higher incidence during 2016 and 2015, two time periods of high incidence. There have been different observations based on gender, where some studies show no difference and others show slight advantages for male or female individuals. The data are so varied that a difference is even found within a specific city, one example being Singapore. ${ }^{51,53-58}$ The literature does not support that greater incidence in children and male individuals is a defined characteristic of dengue. Such contrast demonstrates the importance of local factors that might contribute greater to disease incidence, such as transmission intensity, local herd immunity, and temporal patterns.

The geographic distribution of dengue between the years 2009 and 2017 in Medellín shows varied impact within the city. Higher incidence tends to be found in the center eastern and northeastern of the city, in locations with lower income, lower education rates, high unemployment, and high rates of labor informality-individuals who are not taxed or monitored by the 
2009

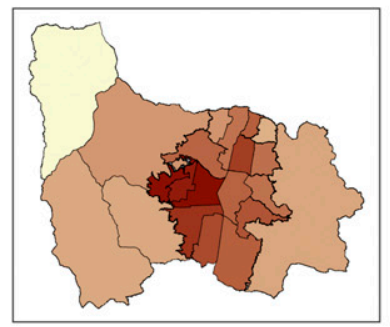

2012

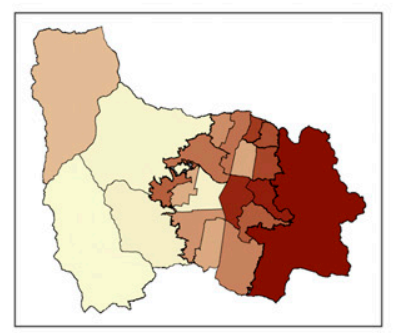

2015

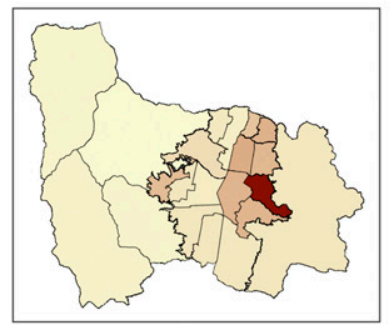

2010

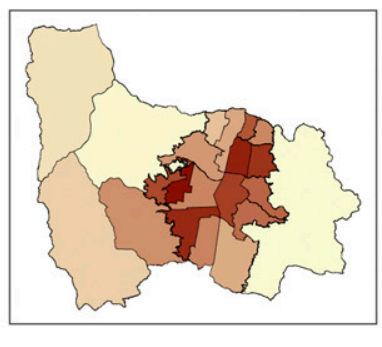

2013
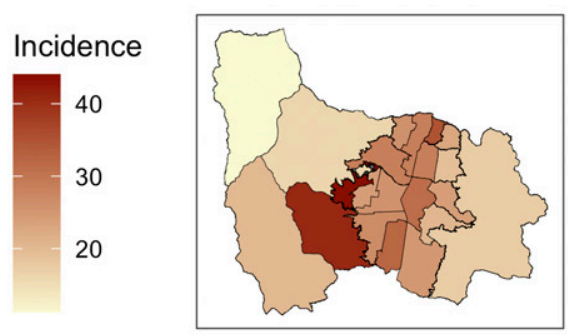

2016


2011

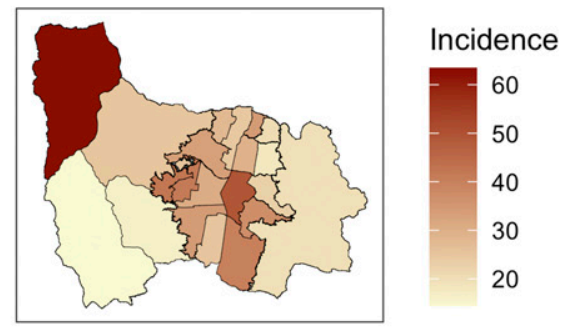

2014


Incidence

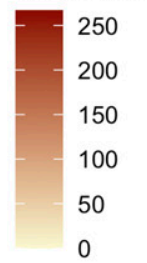

2017
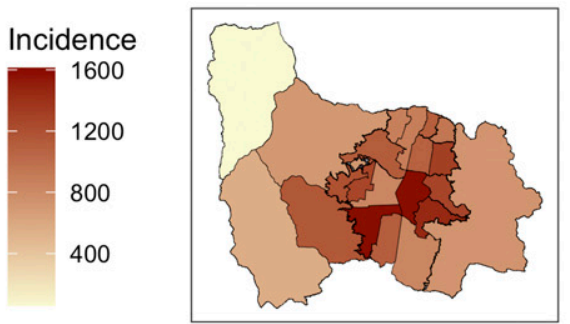

Incidence

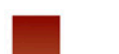

90

60

30

FIGURE 4. Map of dengue disease incidence per 100,000 in Medellin, Colombia, 2009-2017, separated by comuna and corregimiento; legend is scaled to levels within that specific year, 2009-2017. This figure appears in color at www.ajtmh.org.

government. ${ }^{59}$ Dengue follows the same pattern in other locations such as in the province of São Paulo, Brazil, and in Cali, Colombia. ${ }^{44,60,61}$ Denser living conditions in lower grossdomestic product areas could provide more spaces for still water and $A$. aegypti vector to breed in. Our rural to urban comparison loosely supports this hypothesis, where more rural areas generally saw lower incidence of dengue as has been shown in other locations, such as Delhi, India. ${ }^{62}$ Dengue hot spots can also help predict the areas of future outbreaks. In both 2010 and 2016, there was a surge in cases around areas that were shown to have high incidence the year before. In 2009, the areas around San Javier and Aranjuez had the highest incidence in the city, and in 2010, they were the center of the outbreak. In 2015, Villa Hermosa was an outlier in dengue incidence, and in 2016, it continued to have high incidence and was joined by the immediate surrounding comunas. However, this hypothesis requires further analysis and support. Having an active response in areas that show increasing incidence has been effective in containing illnesses. ${ }^{63,64}$ To plan an effective public health program, it is imperative to target the disease strategically, especially one with such widespread movement and impact as dengue.

The peak of dengue cases within our time period was in 2016, right in the middle of the Zika pandemic. There were Zika outbreaks in Nicaragua, Brazil, and Puerto Rico, and cases were confirmed in a total of 45 countries in the Americas. ${ }^{65-69}$ In other parts of Colombia, there were a very high number of
Zika cases as well, supported by the prudent public health department capturing cases around the country and confirming them through RT-PCR, with priority for testing pregnant women. ${ }^{70,71}$ However, although Medellín did see some Zika cases, it was particularly low at an incidence of 22.1/ 100,000 population, though having a high number of dengue cases. Considering similarities in the clinical presentation and IgM cross-reactivity between Zika and dengue viruses, it might be important to look closely to see whether there was any overlap. ${ }^{72}$ Co-circulation of both diseases can be dangerous, and differentiating them with more modern assays can be important to prepare for potential future outbreaks and cross-reactivity.

Having access to such comprehensive data allowed for a basic analysis of epidemiological trends; however, more work is required. If our data were supported fully by $\operatorname{lgM}$ or other techniques such as PCR or paired samples, we would have greater confidence in our results. This is especially important during standard years, where there were a higher percentage of negative cases and when other diseases could be experiencing outbreaks. Pointed epidemiological analysis focused on specific comunas would help identify risk factors and locations that could fuel vector habitability. Further pointed analysis on precipitation trends, individual movement, and phylogenetics would have potentially shown interesting patterns of dengue and helped support suggestions that we made. Serotype data would also help us inform analysis related to severity and case numbers. We hope to encourage 
such analysis in the future and an overall increase in broad epidemiological analysis within cities worldwide, using simple and effective analytical methods.

This study serves as an update on dengue trends in Medellín and provides some potential improvements to disease surveillance and control. This information could aid in public health initiatives to coordinate more effectively within the city and toward certain demographics, bringing down costs and improving efficiency. Having consistent analysis of such a large and important city within Colombia is important to support studies throughout the country and the city itself. Developing countries are strained by the high cost and limited effectiveness of clinical care and diagnostic techniques. The dengue field must prioritize better methods so that interventions and surveillance can be more effective. Public health initiatives supported by our data can lead to cost-effective and comprehensive results, a benefit for the communities overall.

Received October 1, 2019. Accepted for publication January 22, 2021. Published online March 22, 2021.

Note: Supplemental tables and figures appear at www.ajtmh.org.

Acknowledgments: We thank Dr. Thomas Yuill for guidance in the dengue field and promoting a passion for infectious disease research; Leah Katzelnick for helpful comments on the manuscript; members of the Secretariat of Health in Medellín, Colombia, for access to the citywide database and responsiveness with regard to questions about the data organization; Lina Zuluaga and Jennifer Alexandra Montoya for their quality control expertise; and the various health centers involved in collecting the dengue disease data. This work was supported by Programa de Estudio y Control de Enfermedades Tropicales (PECET) at Universidad de Antioquia in Medellín, Antioquia, Colombia. The American Society of Tropical Medicine and Hygiene (ASTMH) assisted with publication expenses.

Authors' addresses: Colin M. Warnes, Eduardo Santacruz-Sanmartín, and Iván Darío Vélez, PECET, School of Medicine, Universidad de Antioquia, Medellin, Colombia, E-mails: cwarnes@brandeis.edu, santacruzsanmartin@gamil.com, and idvelez@pecet-colombia.org. Fausto Bustos Carrillo, Department of Epidemiology, Sustainable Sciences Institute (SSI), San Francisco, CA, E-mail: bustos.fausto@ gmail.com.

\section{REFERENCES}

1. Bhatt $S$ et al., 2013. The global distribution and burden of dengue. Nature 496: 504-507.

2. Soo KM, Khalid B, Ching SM, Chee HY, 2016. Meta-analysis of dengue severity during infection by different dengue virus serotypes in primary and secondary infections. PLoS One 11: e0154760.

3. Katzelnick LC, Gresh L, Halloran ME, Mercado JC, Kuan G, Gordon A, Balmaseda A, Harris E, 2017. Antibody-dependent enhancement of severe dengue disease in humans. Science 358: 929-932.

4. Messina JP et al., 2019. The current and future global distribution and population at risk of dengue. Nat Microbiol 4: 1508-1515.

5. Plennevaux E et al., 2018. Impact of dengue vaccination on serological diagnosis: insights from phase III dengue vaccine efficacy trials. Clin Infect Dis 66: 1164-1172.

6. Vannice KS et al., 2018. Clinical development and regulatory points for consideration for second-generation live attenuated dengue vaccines. Vaccine 36: 3411-3417.

7. Messina JP, Brady OJ, Pigott DM, Golding N, Kraemer MUG, Scott TW, Wint GRW, Smith DL, Hay SI, 2015. The many projected futures of dengue. Nat Rev Microbiol 13: 230-239.

8. Parreira R, Centeno-Lima S, Lopes A, Portugal-Calisto D, Constantino A, Nina J, 2014. Dengue virus serotype 4 and chikungunya virus coinfection in a traveller returning from Luanda, Angola, January 2014. Euro Surveill 19: 20730.

9. Sharma KD, Mahabir RS, Curtin KM, Sutherland JM, Agard JB, Chadee DD, 2014. Exploratory space-time analysis of dengue incidence in Trinidad: a retrospective study using travel hubs as dispersal points, 1998-2004. Parasit Vectors 7: 341.

10. Nunes MRT et al., 2014. Air travel is associated with intracontinental spread of dengue virus serotypes 1-3 in Brazil. PLoS Negl Trop Dis 8: e2769.

11. Siraj AS, Santos-Vega M, Bouma MJ, Yadeta D, Ruiz Carrascal D, Pascual M, 2014. Altitudinal changes in malaria incidence in highlands of Ethiopia and Colombia. Science 343: 1154-1158.

12. Granger D, Leo YS, Lee LK, Theel ES, 2017. Serodiagnosis of dengue virus infection using commercially available antibody and NS1 antigen ELISAs. Diagn Microbiol Infect Dis 88: 120-124.

13. Dussart $P$ et al., 2006. Evaluation of an enzyme immunoassay for detection of dengue virus NS1 antigen in human serum. Clin Vaccine Immunol 13: 1185-1189.

14. Pal S, Dauner AL, Mitra I, Forshey BM, Garcia P, Morrison AC, Halsey ES, Kochel TJ, Wu SJ, 2014. Evaluation of dengue NS1 antigen rapid tests and ELISA kits using clinical samples. PLoS One 9: e113411.

15. Mansfield KL, Horton DL, Johnson N, Li L, Barrett ADT, Smith DJ, Galbraith SE, Solomon T, Fooks AR, 2011. Flavivirus-induced antibody cross-reactivity. J Gen Virol 92: 2821-2829.

16. Rojas A et al., 2018. Internally controlled, multiplex real-time reverse transcription PCR for dengue virus and yellow fever virus detection. Am J Trop Med Hyg 98: 1833-1836.

17. Sanchez JD, https://www.facebook.com/pahowho. OPS/OMS Dengue: Información general. Pan American Health Organization/ World Health Organization. Available at: https://www.paho.org/hq/ index.php?option=com_content\&view=article\&id=4493:2010informacion-general-dengue\&ltemid=40232\&lang=es. Accessed March 21, 2019.

18. Jiménez-Silva CL, Carreño MF, Ortiz-Baez AS, Rey LA, VillabonaArenas CJ, Ocazionez RE, 2018. Evolutionary history and spatio-temporal dynamics of dengue virus serotypes in an endemic region of Colombia. PLoS One 13: e0203090.

19. Restrepo BN, Beatty ME, Goez Y, Ramirez RE, Letson GW, Diaz FJ, Piedrahita LD, Osorio JE, 2014. Frequency and clinical manifestations of dengue in urban Medellin, Colombia. J Trop Med 2014: 872608.

20. Villar LA, Rojas DP, Besada-Lombana S, Sarti E, 2015. Epidemiological trends of dengue disease in Colombia (2000-2011): a systematic review. PLoS Negl Trop Dis 9: e0003499.

21. World Health Organization, 2018. Disease Outbreaks. SEARO. Available at: http://www.searo.who.int/topics/disease outbreaks/ en/. Accessed December 22, 2018.

22. Dirección Nacional de Estadística (DANE), 2019. Proyecciones de población. Available at: https://www.dane.gov.co/index.php/ estadisticas-por-tema/demografia-y-poblacion/proyeccionesde-poblacion. Accessed March 21, 2019.

23. Vázquez EF, Morollón FR, 2013. Defining the Spatial Scale in Modern Regional Analysis: New Challenges from Data at Local Level. Heidelberg, New York, Dordrecht London: Springer Science \& Business Media.

24. Ministerio de Salud y Protección Social de Colombia, 2019. INFORME SITUACIONDEDENGUE.pdf. Available at:https://minsalud.gov.co/ Documentos\%20y\%20Publicaciones/INFORME\%20SITUACION \%20DE\%20DENGUE.pdf. Accessed May 4, 2019.

25. Shepard DS, Undurraga EA, Halasa YA, Stanaway JD, 2016. The global economic burden of dengue: a systematic analysis. Lancet Infect Dis 16: 935-941.

26. Lee JS, 2017. A multi-country study of the economic burden of dengue fever: Vietnam, Thailand, and Colombia. PLoS Negl Trop Dis 11: e0006037.

27. Hadinegoro SRS, 2012. The revised WHO dengue case classification: does the system need to be modified? Paediatr Int Child Health 32: 33-38.

28. Moreno HA, Vélez MV, Montoya JD, Rhenals RL, 2006. La Lluvia y los Deslizamientos de tierra en antioquia: análisis de su ocurrencia en las escalas interanual, intraanual y diaria. Rev EIA 2006: 59-69.

29. Ladner J, Rodrigues M, Davis B, Besson MH, Audureau E, Saba J, 2017. Societal impact of dengue outbreaks: stakeholder perceptions and related implications. A qualitative study in Brazil, 2015. PLoS Negl Trop Dis 11: e0005366.

30. Arango AE, Jaramillo S, Perez J, Ampuero JS, Espinal D, Donado J, Felices V, Garcia J, Laguna-Torres A, 2015. Influenza-like 
illness sentinel surveillance in one hospital in Medellin, Colombia. 2007-2012. Influenza Other Respir Viruses 9: 1-13.

31. Bowman LR, Runge-Ranzinger S, McCall PJ, 2014. Assessing the relationship between vector indices and dengue transmission: a systematic review of the evidence. PLoS Negl Trop Dis 8: e2848.

32. Wilson ME, Chen LH, 2014. Dengue: update on epidemiology. Curr Infect Dis Rep 17: 457.

33. Tan KK, Zulkifle NI, Abd-Jamil J, Sulaiman S, Yaacob CN, Azizan NS, Che Mat Seri NAA, Samsudin NI, Mahfodz NH, AbuBakar S, 2017. Disruption of predicted dengue virus type 3 major outbreak cycle coincided with switching of the dominant circulating virus genotype. Infect Genet Evol 54: 271-275.

34. Rajarethinam J et al., 2018. Dengue in Singapore from 2004 to 2016: cyclical epidemic patterns dominated by serotypes 1 and 2. Am J Trop Med Hyg 99: 204-210.

35. Low JGH et al., 2011. The early clinical features of dengue in adults: challenges for early clinical diagnosis. PLoS Negl Trop Dis 5: e1191.

36. Beltrán-Silva SL, Chacón-Hernández SS, Moreno-Palacios E, Pereyra-Molina JÁ, 2018. Clinical and differential diagnosis: dengue, chikungunya and Zika. Rev Médica Hosp Gen México 81: 146-153.

37. Stewart-lbarra AM et al., 2018. The burden of dengue fever and chikungunya in southern coastal Ecuador: epidemiology, clinical presentation, and phylogenetics from the first two years of a prospective study. Am J Trop Med Hyg 98: 1444-1459.

38. Reller ME, de Silva AM, Miles JJ, Jadi RS, Broadwater A, Walker K, Woods C, Mayorga O, Matute A, 2016. Unsuspected dengue as a cause of acute febrile illness in children and adults in western Nicaragua. PLoS Negl Trop Dis 10: e0005026.

39. Sa-ngamuang C, Haddawy P, Luvira V, Piyaphanee W, lamsirithaworn S, Lawpoolsri S, 2018. Accuracy of dengue clinical diagnosis with and without NS1 antigen rapid test: comparison between human and Bayesian network model decision. PLoS Negl Trop Dis 12: e0006573.

40. Abhishek KS, Chakravarti A, 2019. Simultaneous detection of IgM antibodies against dengue and chikungunya: coinfection or cross-reactivity? J Fam Med Prim Care 8: 2420-2423.

41. Felix AC, Souza NCS, Figueiredo WM, Costa AA, Inenami M, da Silva RMG, Levi JE, Pannuti CS, Romano CM, 2017. Cross reactivity of commercial anti-dengue immunoassays in patients with acute Zika virus infection. J Med Virol 89: 1477-1479.

42. Houghton-Triviño N, Montaña D, Castellanos J, 2008. Dengueyellow fever sera cross-reactivity; challenges for diagnosis. Rev Salud Pública 10: 299-307.

43. Zapata-Vanegas MA, Saturno-Hernández PJ, 2020. Contextual factors favouring success in the accreditation process in Colombian hospitals: a nationwide observational study. BMC Health Serv Res 20: 772.

44. Delmelle E, Hagenlocher M, Kienberger S, Casas I, 2016. A spatial model of socioeconomic and environmental determinants of dengue fever in Cali, Colombia. Acta Trop 164: 169-176.

45. Casas I, Delmelle E, 2019. Landscapes of healthcare utilization during a dengue fever outbreak in an urban environment of Colombia. Environ Monit Assess 191: 279.

46. Castillo-Palacio M, Harrill R, Zuñiga-Collazos A, 2017. Back from the brink. Worldw Hosp Tour Themes 9: 300-315.

47. Vasquez V, Haddad E, Perignon A, Jaureguiberry S, Brichler $S$, Leparc-Goffart I, Caumes E, 2018. Dengue, chikungunya, and zika virus infections imported to Paris between 2009 and 2016: characteristics and correlation with outbreaks in the French overseas territories of Guadeloupe and Martinique. Int J Infect Dis 72: 34-39.

48. Tian E, Sun Z, Faria NR, Yang J, Cazelles B, Huang S, Xu B, Yang $Q$, Pybus OG, Xu B, 2017. Increasing airline travel may facilitate co-circulation of multiple dengue virus serotypes in Asia. PLoS Negl Trop Dis 11: e0005694.

49. Lana RM, Gomes MFDC, Lima TFM, Honório NA, Codeço CT, 2017. The introduction of dengue follows transportation infrastructure changes in the state of Acre, Brazil: a networkbased analysis. PLoS Negl Trop Dis 11: e0006070.

50. Burattini MN, Lopez LF, Coutinho FAB, Siqueira JB, Jr, Homsani S, Sarti E, Massad E, 2016. Age and regional differences in clinical presentation and risk of hospitalization for dengue in Brazil, 2000-2014. Clinics 71: 455-463.
51. Rojas DP et al., 2018. Epidemiology of dengue and other arboviruses in a cohort of school children and their families in Yucatan, Mexico: baseline and first year follow-up. PLoS Negl Trop Dis 12: e0006847.

52. Dhar-Chowdhury P, Paul KK, Haque CE, Hossain S, Lindsay LR, Dibernardo A, Brooks WA, Drebot MA, 2017. Dengue seroprevalence, seroconversion and risk factors in Dhaka, Bangladesh. PLoS Negl Trop Dis 11: e0005475.

53. Byrne AB, Gutierrez GF, Bruno A, Córdoba MT, Bono MM, Polack FP, Talarico LB, Quipildor MO, 2018. Age-associated differences in clinical manifestations and laboratory parameters during a dengue virus type 4 outbreak in Argentina. J Med Virol 90: 197-203.

54. Yew YW, Ye T, Ang LW, Ng LC, Yap G, James L, Chew SK, Goh $\mathrm{KT}, 2009$. Seroepidemiology of dengue virus infection among adults in Singapore. Ann Acad Med Singapore 38: 667-675.

55. Yung CF, Chan SP, Thein TL, Chai SC, Leo YS, 2016. Epidemiological risk factors for adult dengue in Singapore: an 8-year nested test negative case control study. BMC Infect Dis 16: 323.

56. Lee P, Yeung ACM, Chen Z, Chan MCW, Sze KH, Chan PKS, 2018. Age-specific seroprevalence of dengue infection in Hong Kong. J Med Virol 90: 1427-1430.

57. Tomashek KM et al., 2017. Clinical and epidemiologic characteristics of dengue and other etiologic agents among patients with acute febrile illness, Puerto Rico, 2012-2015. PLoS Negl Trop Dis 11: e0005859.

58. Mukhtar MU, Mukhtar M, lqbal N, 2018. Dengue fever is an emerging public health concern in the city of Multan, Pakistan: its seroprevalence and associated risk factors. Microbiol Immunol 62: 729-731.

59. Gallego Ortiz S, González EJM, García GA, 2018. Análisis espacial de la informalidad laboral a nivel intra-urbano. ResearchGate. Available at: https://www.researchgate.net/publication/ 327068663_Analisis_Espacial_de_la_Informalidad_Laboral_a_ Nivel_Intra-urbano. Accessed December 17, 2018.

60. Mondini A, 2009. Spatio-temporal tracking and phylodynamics of an urban dengue 3 outbreak in São Paulo, Brazil. PLoS Negl Trop Dis 3: e448.

61. Farinelli EC, Baquero OS, Stephan C, Chiaravalloti-Neto F, 2018. Low socioeconomic condition and the risk of dengue fever: a direct relationship. Acta Trop 180: 47-57.

62. Vikram Ket al., 2016. An epidemiological study of dengue in Delhi, India. Acta Trop 153: 21-27.

63. Wojda TR, Valenza PL, Cornejo K, McGinley T, Galwankar SC, Kelkar D, Sharpe RP, Papadimos TJ, Stawicki SP, 2015. The ebola outbreak of 2014-2015: from coordinated multilateral action to effective disease containment, vaccine development, and beyond. J Glob Infect Dis 7: 127-138.

64. Farah ZE, Khatib O, Hamadeh S, Ahmad K, El Bazzal B, Zalloua P, Ammar W, Ghosn N, 2018. Containment of highly pathogenic avian influenza $\mathrm{A}(\mathrm{H} 5 \mathrm{~N} 1)$ virus, Lebanon, 2016. Emerg Infect Dis 24: 374-376.

65. Villela DAM, Bastos LS, Carvalho LMD, Cruz OG, Gomes MFC, Durovni B, Lemos MC, Saraceni V, Coelho FC, Codeço CT, 2017. Zika in Rio de Janeiro: assessment of basic reproduction number and comparison with dengue outbreaks. Epidemiol Infect 145: 1649-1657.

66. Gregianini TS, Tumioto-Giannini GL, Favreto C, Plentz LC, Ikuta $\mathrm{N}$, da Veiga ABG, 2018. Dengue in rio grande do sul, Brazil: 2014 to 2016. Rev Med Virol 28: e1960.

67. Li R et al., 2017. Cost-effectiveness of increasing access to contraception during the Zika virus outbreak, Puerto Rico, 2016. Emerg Infect Dis 23: 74-82.

68. Faria NR et al., 2017. Establishment and cryptic transmission of Zika virus in Brazil and the Americas. Nature 546: 406-410.

69. Zambrana JV et al., 2018. Seroprevalence, risk factor, and spatial analyses of Zika virus infection after the 2016 epidemic in Managua, Nicaragua. Proc Natl Acad Sci U S A 115: 9294-9299.

70. Pacheco $O$ et al., 2020. Zika virus disease in Colombia - preliminary report. N Engl J Med 383: e44.

71. Alvarez Castaño VH, Guzmán Rodríguez SL, Moreno Segura CM, Trujillo González ÁA, 2017. Indicadores Básicos de Salud 2016. Alcaldía de Medellín, Colombia: Secretaría de Salud.

72. Steinhagen K et al., 2016. Serodiagnosis of Zika virus (ZIKV) infections by a novel NS1-based ELISA devoid of cross-reactivity with dengue virus antibodies: a multicohort study of assay performance, 2015 to 2016. Eurosurveillance 21: 30426. 